\title{
Study of breakup mechanism of a loosely bound projectile in a region of Coulomb-breakup dominance
}

\author{
H. Okamura ${ }^{1, a}$, K. Hatanaka ${ }^{1}$, T. Ikeda ${ }^{2}$, K. Itoh ${ }^{2}$, H. Kumasaka ${ }^{2}$, Y. Maeda ${ }^{3}$, T. Saito ${ }^{4}$, H. Sakai ${ }^{4}$, K. Sekiguchi $^{5}$, \\ K. Suda ${ }^{5}$, R. Suzuki ${ }^{2}$, T. Uesaka ${ }^{6}$, T. Wakasa ${ }^{7}$, and K. Yako ${ }^{4}$ \\ 1 Research Center for Nuclear Physics (RCNP), Ibaraki, Osaka 567-0047, Japan \\ 2 Department of Physics, Saitama University, Saitama 338-8570, Japan \\ 3 Department of Applied Physics, Miyazaki University, Miyazaki, Miyazaki 889-2192, Japan \\ 4 Department of Physics, University of Tokyo, Bunkyo, Toyo 113-0033, Japan \\ 5 RIKEN Nishina Center, Wako, Saitama 351-0198, Japan \\ 6 Center for Nuclear Study (CNS), University of Tokyo, Bunkyo, Toyo 113-0033, Japan \\ 7 Department of Physics, Kyushu University, Higashi, Fukuoka 812-8581, Japan
}

\begin{abstract}
A kinematically complete measurement of the deuteron elastic break reaction has been carried out at $E_{d}=140 \mathrm{MeV}$ on ${ }^{12} \mathrm{C},{ }^{40} \mathrm{Ca},{ }^{90} \mathrm{Zr}$, and ${ }^{208} \mathrm{~Pb}$ targets in a wide angular range of $\theta_{p}, \theta_{n}=0^{\circ}-8^{\circ}$ for a detailed study of the reaction mechanism of loosely bound projectiles in kinematical regions of Coulomb-breakup dominance. A finite-range calculation of the post-form distorted-wave Born approximation is presented.
\end{abstract}

\section{Introduction}

Studies of Coulomb-breakup of composite nuclei have been of constant interest in nuclear physics. The recent discovery of very extended halo nuclei has generated renewed interest in methods for the calculation of Coulomb-breakup. It also has attracted attention in relation to the astrophysical interest. While an exciting application is in the study of such exotic nuclei, the low intensity currently available of secondary beams of these new nuclear species means that present data carry significant experimental uncertainty and are still very limited. Such data inevitably do not challenge sufficiently between different theoretical descriptions.

In the previous study $[1,2]$, we proposed to pursue the problem by using a light-ion primary beam. The deuteron can be considered as the simplest example of an "exotic" nucleus, because of its small breakup-energy and the dominance of the $S$-wave in the ground state having no ambiguity in the wave-function. The $(d, p n)$ elastic breakup was measured at $\theta_{p}=\theta_{n}=0^{\circ}$ and at $E_{d}=56,140$, and $270 \mathrm{MeV}$ on various targets ranging from $Z=6$ to 82 . The data were expected to provide a ground for critical tests of proposed various models. However, the angular resolution was found to be insufficient for the data to be directly compared with theoretical calculations, which predict steep and complicated angular distributions at $\theta \simeq 0^{\circ}$. Calculations also suggest a need to measure the angular correlation up to fairly large angles. These requirements strongly motivated us to carry out the present experiment for extended and precise studies of the reaction mechanism with Coulomb-breakup dominance.

\footnotetext{
a e-mail: okamura@rcnp.osaka-u.ac.jp
}

\section{Experiment}

Due to the use of quadrupole-dipole-type magnetic spectrometer in the previous experiments $[1,2]$, the proton an-

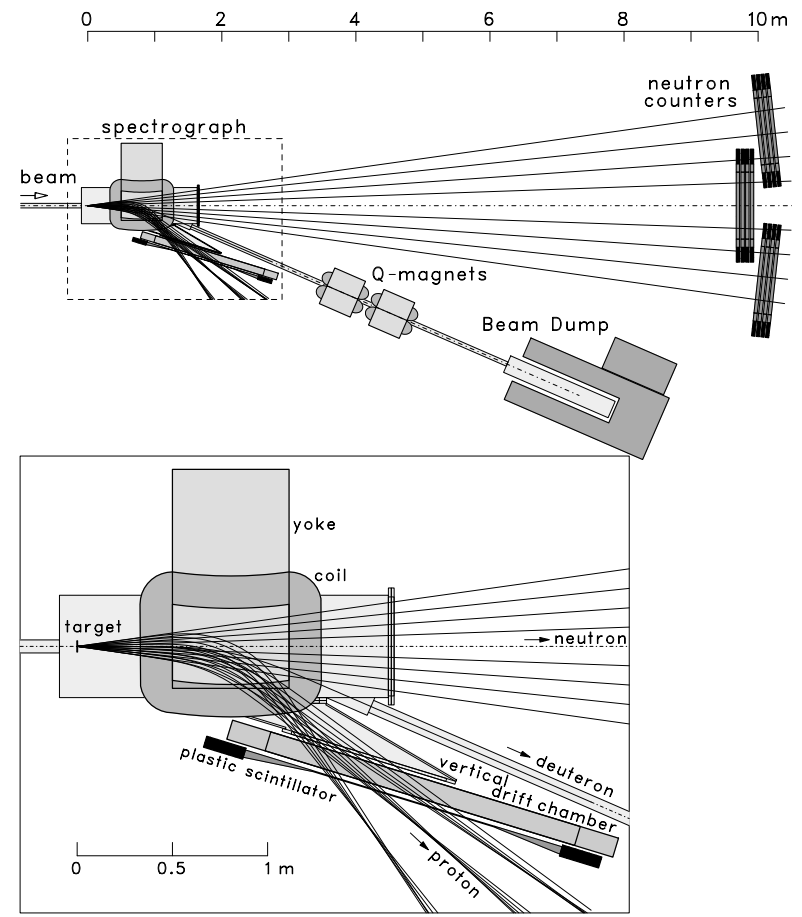

Fig. 1. Schematic layout of the experimental setup constructed at the RCNP ESS course, together with typical particle-trajectories. 
gular resolution was seriously deteriorated and the neutron angle was fixed only at $0^{\circ}$. In the present experiment, a new beam-line has been constructed in the E experimental area at RCNP (Fig. 1) with a simple spectrometer system consisting of a bending magnet and the vertical drift chamber. Both protons and neutrons can be measured up to $8^{\circ}$ with a resolution of $0.5^{\circ}$ (FWHM) or better. The data have been re-measured only at $E_{d}=140 \mathrm{MeV}$ because the proton energy range is limited to $50-110 \mathrm{MeV}$ by the spectrometer. The neutron detection efficiency has been calibrated by using the ${ }^{7} \mathrm{Li}(p, n)^{7} \mathrm{Be}$ reaction, accelerating $\mathrm{H}_{2}^{+}$, the singlycharged hydrogen molecule, with the same cyclotron magnetic field as that for the deuteron in order to minimize the time lag between the calibration and the breakup measurement and thus minimize the systematic uncertainties.

\section{Results}

Figure 2 shows the coplanar angular correlations of the proton energy spectra obtained for the $(d, p n)$ elastic breakup reaction on ${ }^{12} \mathrm{C},{ }^{40} \mathrm{Ca},{ }^{90} \mathrm{Zr}$, and ${ }^{208} \mathrm{~Pb}$ targets. The angular bin widths are $1^{\circ}$ both for $\theta_{p}$ and $\theta_{n}$ except at $\theta_{p}=\theta_{n}=0^{\circ}$, where steep angular distributions are expected thus the bin widths are chosen to be $0.5^{\circ}$. It is remarkable that the spectra are well scaled among lighter targets; the shapes are almost identical between ${ }^{12} \mathrm{C}$ and ${ }^{40} \mathrm{Ca}$ and very similar for ${ }^{90} \mathrm{Zr}$, but the distribution drastically changes for ${ }^{208} \mathrm{~Pb}$. The double-peak structure at $\theta_{p}=\theta_{n}=0^{\circ}$ with a sharp dip at $E_{p}=E_{n}$, where the $p$ - $n$ relative energy becomes 0 , indicates a large contribution from $P$-wave breakup, presumably resulting from the Coulomb breakup dominance. At the same time, the asymmetric spectrum shape suggests contributions from higher partial waves, post-acceleration effects, and/or interference with the nuclear breakup. A "pure-Coulomb" calculation in the framework of adiabatic approximation [3], for example, roughly accounts for the tendency of data but does not reproduce the distinctive difference between ${ }^{208} \mathrm{~Pb}$ and other targets. This time the data should be truly useful to establish a theoretical treatment of Coulomb-breakup of loosely bound projectiles.

\section{Analysis}

A simple analysis has been made for understanding the behavior of the spectra. This kind of data are commonly analyzed by using the continuum-discretized coupled-channels method (CDCC) [4]. However, if the Coulomb interaction is most important, it is natural to treat the problem in the post-formalism than in the prior-formalism, which is employed in CDCC, and may be likely to justify to treat the remaining nuclear interactions perturbatively. In fact, the post-form distorted-wave Born approximation (DWBA) is successfully used for the analysis of $(d, p n)$ breakup data near the Coulomb-barrier region [5]. Since the triple integration is a bit involved, the zero-range approximation is employed in those analyses, which unfortunately is not justified in the present case where $k_{d}=3.8 \mathrm{fm}^{-1}$ at $140 \mathrm{MeV}$.
A finite-range calculation has been carried out by expanding the distorted-waves with Coulomb wave-functions, in a similar manner to the plane-wave expansion [6] employed in the code DwUck 5 [7]. The post-form DWBA $T$-matrix is given by

$$
\begin{aligned}
T_{\mathrm{DW}}= & \left\langle\chi_{n}\left(\mathbf{k}_{n}\right) \chi_{p}\left(\mathbf{k}_{p}\right)\left|V_{n p}\right| \varphi_{d} \chi_{d}\left(\mathbf{k}_{d}\right)\right\rangle \\
= & \iiint d \mathbf{q}_{n} d \mathbf{q}_{p} d \mathbf{q}_{d} /(2 \pi)^{9} \\
& \times\left\langle\chi_{n}\left(\mathbf{k}_{n}\right) \mid \phi_{n}\left(\mathbf{q}_{n}\right)\right\rangle\left\langle\chi_{p}\left(\mathbf{k}_{p}\right) \mid \chi_{p}^{\mathrm{C}}\left(\mathbf{q}_{p}\right)\right\rangle\left\langle\chi_{d}^{\mathrm{C}}\left(\mathbf{k}_{d}\right) \mid \chi_{d}\left(\mathbf{q}_{d}\right)\right\rangle \\
& \times\left\langle\phi_{n}\left(\mathbf{q}_{n}\right) \chi_{p}^{C}\left(\mathbf{q}_{p}\right)\left|V_{n p}\right| \varphi_{d} \chi_{d}^{C}\left(\mathbf{q}_{d}\right)\right\rangle .
\end{aligned}
$$

The last term is the "pure-Coulomb" $T$-matrix, where only the $n-p$ nuclear interaction $\left(V_{n p}\right)$ and the Coulomb interactions are taken into account. By introducing the localmomentum approximation (LMA), it is separated into the projectile form factor and the Bremsstrahlung $T$-matrix,

$$
\begin{aligned}
T_{\mathrm{DW}}^{\mathrm{pC}} & =\left\langle\phi_{n}\left(\mathbf{q}_{n}\right) \chi_{p}^{C}\left(\mathbf{q}_{p}\right)\left|V_{n} p\right| \varphi_{d} \chi_{d}^{C}\left(\mathbf{q}_{d}\right)\right\rangle \\
& \simeq\left\langle\phi_{n p}\left(\mathbf{k}_{n p}\right)\left|V_{n p}\right| \varphi_{d}\right\rangle\left\langle\phi_{d}\left(\xi \mathbf{k}_{n}\right) \chi_{p}^{\mathrm{C}}\left(\mathbf{k}_{p}\right) \mid \chi_{d}^{\mathrm{C}}\left(\mathbf{k}_{d}\right)\right\rangle,
\end{aligned}
$$

the latter part of which is readily calculated by using an analytical form [8]. It should be noted that the same form of the "pure-Coulomb" breakup is also obtained starting from the adiabatic approximation [3] instead of the post-form DWBA. The validity of LMA is discussed in ref. [9] by comparison with exact calculations for "pure-Coulomb" breakup. According to this result, the discrepancy is negligibly small for the deuteron breakup, although it becomes larger for heavier systems such as ${ }^{11} \mathrm{Be}$.

The results of calculation are presented in Fig. 2; blue lines are for the "pure-Coulomb" calculation, while red lines are for the full calculation. The same nuclear optical potentials are employed as those in ref. [2], which are consistent with the newer global-potentials for nucleons [10] and the deuteron [11]. The energy-dependence of the potential is taken into account for the ejectiles, $p$ and $n$.

The "pure-Coulomb" calculation accounts for the data at $\theta_{p}=\theta_{n}=0^{\circ}$ fairly well, not only for the double-peak structure but also for the amplitude. It is also fairly successful at $\theta_{p} \simeq \theta_{n}$ and $\phi_{n p}=180^{\circ}$, where protons and neutrons are emitted to the opposite side thus the momentum transfer is small, but seriously underestimate the amplitude at other (particularly backward) angles. By including nuclear interaction, on the other hand, the agreement at (neutron) backward angles is remarkably improved. The nuclear interaction also makes the double-peak structure at forward angles asymmetric, presumably by interference with the Coulomb breakup. However, the shape is much too asymmetric to be compared with the data, indicating the overcontribution from the nuclear interaction. This may be ascribed to the perturbative treatment of $V_{n p}$, in other words, effects of $n-p$ final-state interaction (FSI). This can be understood like the following; the (true) three-body scattering wave function will be written as

$$
\Psi_{n p A}=\chi_{n} \chi_{p}\left(1+\psi_{n p}^{\mathrm{FSI}}+\cdots\right)
$$


$19^{\text {th }}$ International IUPAP Conference on Few-Body Problems in Physics
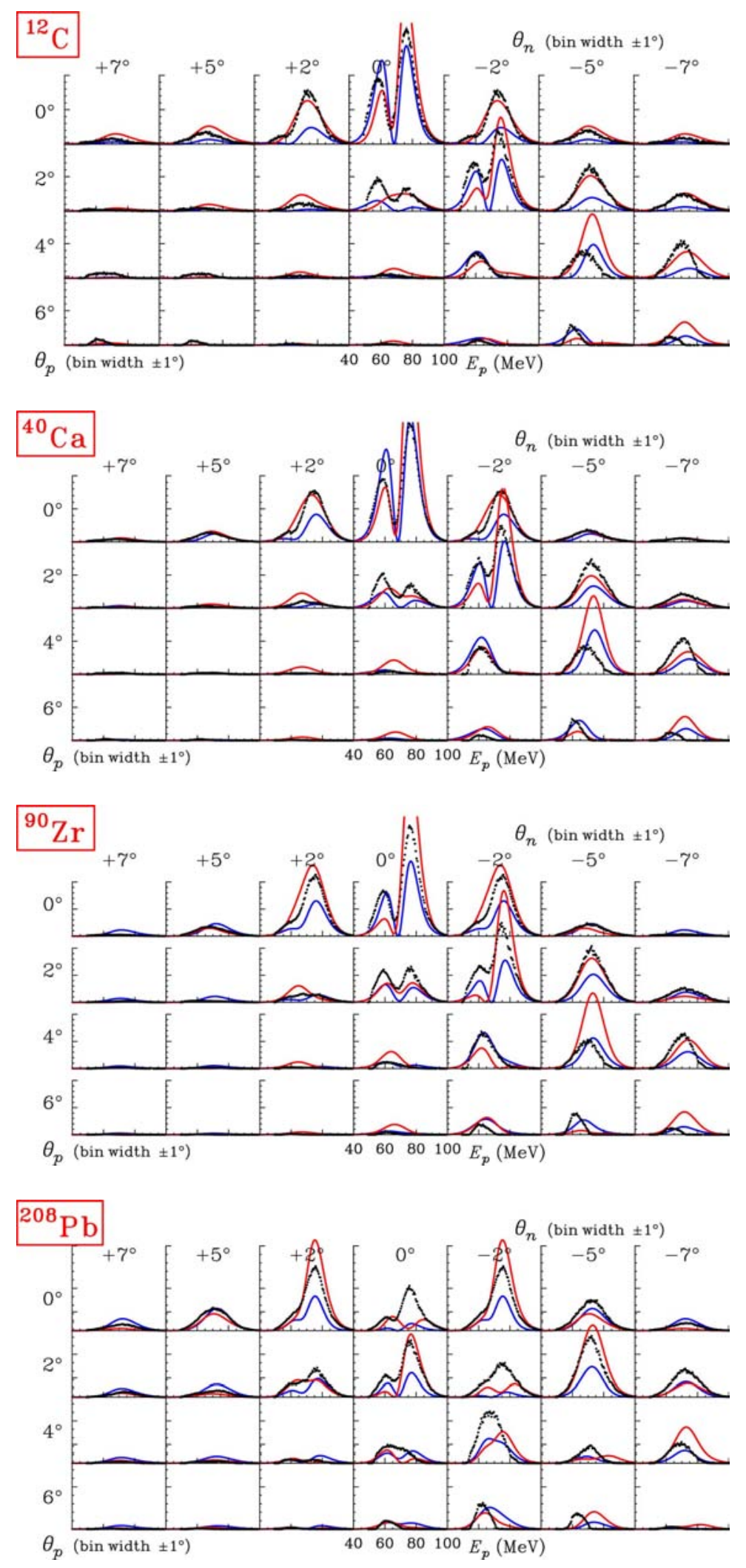

Fig. 2. Proton energy spectra of the $(d, p n)$ elastic breakup reaction at $E_{d}=140 \mathrm{MeV}$ on ${ }^{12} \mathrm{C},{ }^{40} \mathrm{Ca},{ }^{90} \mathrm{Zr}$, and ${ }^{208} \mathrm{~Pb}$ targets. Blue lines are the results of "pure-Coulomb" calculation, and red lines are those of the full calculation including the nuclear potentials. 
where the $n-p$ FSI term $\psi_{n p}^{\mathrm{FSI}}$ is expected to contribute destructively to the $T$-matrix considering the orthogonality between the bound and unbound $n-p$ states,

$$
\left\langle\Psi_{n p A} \mid \varphi_{d} \chi_{d}\right\rangle=0
$$

Further analyses are required to fully understand the whole spectra, which will hopefully lead to development of an efficient and reasonably realistic method to treat the Coulombbreakup reaction of loosely bound projectiles.

\section{References}

1. H. Okamura et al., Phys. Lett. B 325 (1994) 308.

2. H. Okamura et al., Phys. Rev. C 58 (1998) 2180.

3. J.A. Tostevin et al., Phys. Rev. C 57 (1998) 3225; and references therein.

4. N. Austern et al., Phys. Rep. 154 (1987) 125.

5. G. Baur and D. Trautmann, Phys. Rep. 25C (1976) 1525; and references therein.

6. L.A. Charlton, Phys. Rev. C 8 (1973) 146.

7. P.D. Kunz, computer code DwUCK 5 (unpublished).

8. A. Nordsieck, Phys. Rev. 93 (1954) 785.

9. M. Zadro, Phys. Rev. C 66 (2002) 034603.

10. A.J. Koning and J.P. Delaroche, Nucl. Phys. A $\mathbf{7 1 3}$ (2003) 231.

11. H. An and C. Cai, Phys. Rev. C 73 (2006) 054605. 